\title{
P02-2-2 Poster session
}

\section{The inhibitory mechanisms of methylcobalamin on mechanical allodynia in mice infected with herpes simplex virus type-1}

\author{
Takashi Kikukawa, Daisuke Uta, Tsugunobu Andoh \\ Department of Applied Pharmacology, University of Toyama, Japan
}

Herpes zoster is caused by the reactivation of latent varicella-zoster virus (VZV) in the sensory ganglia. When VZV is reactivated, the dermatomal rash and severe dysesthesia (e.g. allodynia) are developed. Furthermore, some patients complain postherpetic neuralgia (PHN) which is induced after healing the rash. However, the existing analgesics are difficult to control these neuropathic pain. Therefore, new analgesics are need to be developed. Methylcobalamin $(\mathrm{MeCbl})$ is an analog of vitamin B12 and used for several neuropathic pain. In this study, we examined whether MeCbl could attenuate herpes zoster-associated pain in mice.

In this study, we used female C57BL/6j mice. Herpes simplex virus-1 (HSV-1), which is a member of the Herpesviridae family as same as VZV, was inoculated topically on the scarified skin of right femoral region. Dynamic mechanical allodynia was evaluated using the paint brush. MeCbl was administered subcutaneously once daily from day 5 after HSV-1 infection. Distribution of peripheral sensory nerve fibers in the skin of footpad, activation of microglia and production of pro-inflammatory cytokines mRNA in spinal cord were analyzed by using immunohistochemical staining or real-time polymerase chain reaction.

Herpes zoster-like skin lesion developed from day 5 post-inoculation, peaked on day 7, and was completely healed by day 20. Dynamic mechanical allodynia was gradually increased from day 3 post-inoculation, peaked on day 7 , and the allodynia was continuously observed after rash healing (PHN). Repetitive administration of MeCbl from day 5 postinoculation inhibited HSV-1-induced mechanical allodynia and C-fiber reduction in the skin of footpad. In spinal cord, HSV-1 infection increased and activated microglia in spinal cord. In addition, the expression of pro-inflammatory cytokines mRNA in spinal cord was also increased by the HSV-1 infection. Repetitive administration of MeCbl inhibited these increases.

In conclusion, these results suggest that $\mathrm{MeCbl}$ is effective for inhibiting not only HSV-1-induced allodynia but also the transition to PHN. In addition to the protection of sensory neurons, the inhibition of both activation of microglia and expression of pro-inflammatory cytokines may be involved in anti-allodynic mechanisms of MeCbl. 